\title{
INVESTIGACIONES
}

\section{Desarrollo docente y diseño curricular en educación superior: una sinergia necesaria para mejorar la calidad de la educación}

\author{
Academic development and curricular design in tertiary education: \\ a necessary synergy for enhancing quality in education
}

\author{
Roberto Espejo Leupin ${ }^{a}$, Verónica Romo López $z^{b}$, Karin Cárdenas ${ }^{c}$ \\ ${ }^{a}$ Director de Enseñanza-Aprendizaje, Universidad Central de Chile. \\ roberto.espejo@gmail.cl \\ ${ }^{b}$ Directora de Efectividad Docente en la Universidad Central de Chile \\ y miembro de Educación en Derechos humanos de Amnistía Internacional Chile. \\ eromo@ucentral.cl \\ ${ }^{c}$ Directora General Académica, Universidad Central de Chile. \\ karin.cardenas@ucentral.cl
}

\begin{abstract}
Se indaga en las posibilidades que otorga para el desarrollo profesional docente de educación superior, la participación en procesos de diseño o rediseño curricular. Se busca conocer esta incidencia desde las percepciones de los y las docentes. Desde la Teoría Fundamentada se analizan 11 entrevistas aplicadas a docentes que han tenido participación en procesos curriculares. Los resultados señalan que esta participación tiene gran incidencia en la forma en que los y las docentes reflexionan respecto de sus prácticas y generan cambios en ellas. Al no encontrar estudios previos referidos a esta posible incidencia, no se discute la transferencia de estos hallazgos. Es destacable la fuerte emergencia de categorías referidas al diálogo que se genera entre colegas y que incide en el cambio y calidad del proceso educativo, transformándose la participación curricular, en un des-aprendizaje que desmonta ideas pre concebidas y facilita la apertura a nuevas posibilidades de prácticas educativas.
\end{abstract}

Palabras claves: educación superior, práctica docente, desarrollo docente, modelo curricular.

\begin{abstract}
The study looks for evidences referred to the possibilities of teacher development due to the participation of them in curriculum design or re-design processes. Interviews were applied to eleven teachers who have this participation. Through Grounded Theory, interviews were analyzed. The results indicate that participation in the processes of curriculum design and re-design have a great impact on the way in which teachers reflect on their teaching practices and generate changes in them. Not having previous studies related to this possible incidence, transfer of these findings are not discussed. It is worth highlighting the strong emergence of categories related to the dialogue that is generated among colleagues and that affects the change and quality of the educational process, because of their transformation through de-learning processes that allow dismantling pre-conceived ideas and opening up new possibilities for educational practices.
\end{abstract}

Key words: higher education, teaching practice, teacher's development, curricular model. 


\section{INTRODUCCIÓN}

González y Espinoza (2008) destacan la necesidad de las universidades e instituciones de educación superior de cautelar la calidad de su gestión en los más diversos aspectos. En cuanto a la gestión de la docencia, los autores sostienen que es necesario incorporar un valor extra a la experiencia que vivencian en su formación los/as estudiantes, lo que implica disponer de ambientes apropiados, de un currículo apropiado, de una investigación relevante en torno a la docencia. Así también, en el estudio de Castro Estrada, Rodríguez Rejas y Urteaga Urías, en México (2016), se sostiene que los/as docentes deberán evidenciar interés en actualizar sus conocimientos para transformarse en educadores superiores capaces de transformar a sus estudiantes. Esta sería la forma de generar, en la época actual, verdadera calidad en el quehacer más relevante de las instituciones de educación superior: la formación y transformación de sus estudiantes para aportar a la mejora de la realidad. En Chile, Salazar Botello, Chiang Vega \& Muñoz Jara (2016), sostienen que las competencias de los y las docentes de educación superior deben ir más allá de la experticia disciplinar y destacan competencias relativas a la generación de un clima de aula apropiado para el aprendizaje y competencias para mediar los aprendizajes de estudiantes diversos. Ya en 2003, Veiravé y Ojeda, planteaban que el docente, para desarrollar educación de calidad, debía ser activo transformador y constructor del currículo. De otra forma no podría identificar los elementos centrales y claves de su práctica profesional. No desarrollan, sin embargo, un estudio de las percepciones que los y las docentes tienen al respecto ni evalúan incidencias en el aula. González Maura \& López Rodríguez (2017) sostienen que las competencias profesionales deben desarrollarse en una instancia participativa, lo que podría implicar la necesaria presencia en el currículo del programa en el que se insertan. Estudios como el de Casanova (2012) y el de Arteaga (2015), señalan la necesidad de considerar en forma sistémica el diseño curricular como forma de asegurar la calidad de la educación; ello sin duda implica considerar al docente. Cabe entonces preguntarse si esta participación docente en los procesos de diseño curricular tiene una incidencia desde el proceso de diseño hacia la práctica profesional docente. Considerando la necesaria búsqueda de la calidad del ejercicio de la docencia en educación superior, se aprecia como interesante el análisis de esta posible incidencia. No se aprecian en los estudios revisados un análisis de la forma en que la participación activa de los y las docentes en el diseño curricular de la carrera en la que laboran, podría generar una mejora de las prácticas educativas y por ende de la calidad del proceso de enseñanza aprendizaje. Por tanto, la pregunta central de la búsqueda que se intenta en esta investigación es:

¿Cuáles son las percepciones de las y los docentes que han participado en procesos de diseño o rediseño curricular, respecto a la incidencia de esta participación en su desarrollo como docente universitario?

\section{ALGUNOS ANTECEDENTES TEÓRICOS Y OBJETIVOS DEL ESTUDIO}

La pregunta por la formación de los profesores es de larga data, y hoy en día se liga directamente con la idea de la calidad educativa, concepto que - dicho sea de paso- no tiene una definición muy precisa. Dicha formación puede tomar muchas formas y dedicarse a distintos niveles de enseñanza. Las primeras iniciativas de formación de profesores 
se orientan a la enseñanza primaria -como en el caso de La Salle- y posteriormente se extienden hacia la enseñanza secundaria. Las y los profesores de secundaria se diferencian de las y los profesores universitarios sólo hacia finales del siglo 19 y comienzos del 20, ya que antes se habría tratado de universitarios o clérigos que poseían una "licencia para enseñar" entregada por una universidad o el mismo papado. En este ámbito la historia de la pedagogía nos muestra una serie de "clásicos" importantes, como por ejemplo la labor pedagógica y humanizadora de un Comenius (Comenius, 2012; Habl, 2017), sustentada en una visión antropológica comprometida con los valores del ser humano y en el acceso para todos y todas a la educación, así como los esfuerzos de educación popular y de formación pedagógica de maestros y maestras de un Pestalozzi (Abbagnano y Visalberghi, 2016; Pestalozzi, 2003).

Posteriormente a esto su formación sigue modelos distintos, en función de los distintos países a los que pertenezcan (Dussel, 2001). Así, la expansión de la enseñanza secundaria, antes reservada sólo a las élites, hace necesario contar con docentes preparados para esto. De ahí el surgimiento de sistemas de formación para los docentes de este nivel.

Desde mediados del siglo pasado se ha observado un fenómeno similar con la enseñanza en Educación Superior. La formación de las y los profesores de este nivel en temas específicos de la docencia, han tomado un lugar importante dentro de los discursos educativos de varios países y presenta desafíos que le son propios, tal como lo exponen Villalobos y Melo (2008) y Aramburuzavala, Hernández-Castilla \& Uribe (2013). Según estos autores, hay diferentes modelos teóricos que guían la Formación del Profesorado Universitario (FPU), pero todos se entienden y caracterizan por ser, con más o menos énfasis, un proceso de estructuración y restructuración de conocimientos que incide en la calidad de la formación de los estudiantes.

Por otra parte, este fenómeno de la búsqueda del desarrollo docente o FPU, como señalan Imbernon \& Guerrero (2017), se asocia con cambios tales como la nueva concepción del trabajo, el papel de la Universidad en la escolarización democrática de la sociedad, la aparición del discurso de las competencias, el acceso cultural masivo, el uso de la tecnología, la idea del lifelong learning y la necesidad de hacer frente a un nuevo tipo de estudiantes que llega a las aulas universitarias.

Esta situación se evidencia al observar la existencia de estructuras dedicadas a la docencia y en particular al desarrollo de profesores y profesoras en este ámbito en las más variadas universidades. Si bien es cierto su forma cambia de una institución a otra, y también el lenguaje utilizado no es homogéneo, la necesidad de estos espacios es más que evidente. Por otra parte, existe evidencia empírica del impacto positivo que estas acciones tienen sobre la labor de los docentes: Gibbs \& Coffer (2004); Chalmers \& Garnier (2015). En Chile, Navarro, Varas, González \& Catalán (2017) analizan la forma en que esta participación es percibida por los/as docentes como relevante para ellos y ellas y la institución, identificando como relevante la implementación de comunidades de aprendizaje.

Dado que en la Educación Superior cumplen la función de profesores/as profesionales que son expertos en sus respectivas áreas, pero no necesariamente en el ámbito educativo, se hace necesario considerar el desarrollo de competencias que les permitan implementar procesos de enseñanza-aprendizaje eficaces y efectivos con los estudiantes que tienen a cargo. Así, Evans (2002) define el desarrollo docente (teacher development) utilizando las ideas de profesionalismo y profesionalidad, considerando que su distinción no siempre 
es clara pero que se relaciona por una parte con actitudes y por otra con las competencias específicas para realizar una tarea.

En este sentido, Imbernón y Guerrero (2017, pp. 5-6) describen un perfil profesional de un docente universitario, a partir de tareas como ser un mediador, conocedor de su disciplina, planificador curricular y tener una actitud colegial para trabajar en equipo.

La participación de los y las docentes en un comité curricular y en particular en el proceso de diseño o rediseño se relaciona con lo señalado por estos autores. Asimismo, es importante considerar la componente política que tiene el trabajo curricular, la que se cruza evidentemente con la temática del poder al exterior y al interior de la organización (Apple, 2012, 2019; Freire, 1958).

En esta línea, Gimeno Sacristán y Pérez (1996) (citados por Osorio, 2017) definen el currículo como un complejo fenómeno que forma parte de múltiples prácticas, todas las cuales inciden en las prácticas educativas. En este fenómeno tienen su espacio e incidencia los contextos institucionales y de aula, el contexto social, histórico de la institución y sin duda el contexto político en el que se manifiestan las formas de autoridad y manejo del poder en la toma de decisiones referidas al currículo y la práctica educativa. El ámbito de la actividad político-administrativa regula, sobre la base de valores e ideologías, el quehacer curricular.

Es en esta mirada política del fenómeno del diseño curricular, que es posible entender el compromiso y la motivación de las personas en dicho proceso. Parafraseando a Freire (1958), este proceso curricular, que involucra la acción de la comunidad, incidirá en la misma, sea para mantenerla, para modificarla o transformarla. Es allí donde se aprecia su contenido político, su sustento teórico e ideológico que le lleva a determinar sus fines y a identificar sus métodos. Y es en la posibilidad de tomar conciencia y participar en la definición de los fines que perseguimos y podemos lograr en una propuesta curricular, lo que permite apropiarse del proceso e intentar vivirlo en plenitud.

Es entonces importante conocer las percepciones de las y los participantes, en estos espacios, para teorizar el mecanismo que permite que su participación se transforme en parte del proceso de desarrollo docente, con el fin de entenderlo mejor y así poder potenciarlo al interior de las instituciones universitarias.

En el ámbito del diseño del currículo, Icarte y Labate (2016) plantean etapas para conducir un diseño curricular basado en competencias, y concluyen que es factible y deseable seguir ciertas etapas definidas por autores diversos y que se requiere que el cuerpo docente se involucre profundamente con el proceso y con la metodología propuesta al interior del mismo, ya que, de lo contrario, la aplicación de la propuesta quedará sólo en un ámbito declarativo.

El diseño del currículo, de acuerdo con Fonseca y Gamboa (2017), es una necesidad que permite otorgar al proceso una dirección y un rasgo de cientificidad que facilita la evaluación de los logros que se proponen. Confirma esta idea Salas (2016) quien determina que la evaluación, aun cuando puede considerarse una etapa final del diseño del currículo, debe estar presente en forma permanente para asegurar lo que todo currículo busca: una formación humana de calidad. Cualquiera sea la definición que se adopte de currículo, es claro que se debe establecer cuál es el tipo de persona que se desea formar y la sociedad que se desea colaborar a construir. Salas (2016) plantea que el diseño curricular de calidad ha de ofrecer respuestas a la formación de los y las estudiantes, tanto para su vida plena como para su inserción crítica en la sociedad. Carr y Kemmis (1988), citados por Casanova (2012), 
consideran que la calidad del currículo y su proceso de implementación está estrechamente relacionada con la práctica del profesorado, que ha de promover el aprendizaje integral del y la estudiante. No sólo se valoran sus adquisiciones de contenidos y habilidades, sino también sus actitudes, pensamiento crítico y su compromiso social y existencial. Por tanto, los autores citados destacan la excelencia del currículo como paralela a la excelencia del acto de enseñar o interacción didáctica, situando por tanto la construcción curricular y la enseñanza en el paradigma socio crítico. Casanova (2012) analiza con fuerza la evaluación del logro de competencias en los y las estudiantes, para determinar la calidad del diseño curricular. Así también Icarte y Labate (2016), buscan una metodología para la revisión o rediseño del currículo en universidades que trabajan con competencias, especificando algunas etapas a desarrollar.

No se aborda en ninguno de los estudios revisados, la percepción que los y las docentes tienen respecto a su involucramiento en el proceso de diseño curricular. Parece posible apreciar que la participación y compromiso del y la docente en este diseño es fundamental para contribuir al proceso formativo estudiantil.

El estudio busca entonces describir e interpretar las percepciones de un grupo de docentes universitarios, respecto de su participación en diseños o rediseños curriculares y en la calidad de su práctica educativa.

Para esto se identifican las percepciones de los actores respecto de las etapas del proceso de diseño curricular que permitirían generar cambios en sus prácticas docentes y las mejoras que los actores eventualmente percibirían en éstas.

\section{METODOLOGÍA}

La metodología utilizada en este proceso corresponde a un paradigma de corte cualitativo. Silverman D., en su texto "Doing Qualitative Research: A Practical Handbook" (citado por Vivar et al., 2013) sostiene que este paradigma busca la profundidad en la comprensión de percepciones de los/as entrevistados/as. Dentro de este enfoque paradigmático, se opta por el diseño y análisis propuestos en la Teoría Fundamentada (Strauss y Corbin, 2002), la cual, de acuerdo con Vivar, Aratzamendi, López y Gordo (2010), permite indagar sobre fenómenos no bien conocidos, para profundizar en el conocimiento de esa realidad y fenómenos específicos. En este sentido es aconsejable la utilización de este diseño para colaborar en la comprensión de las experiencias y significados que los /as participantes de la experiencia o fenómeno, les asignan para su comprensión y asimilación. Así, resulta particularmente recomendable al analizar fenómenos en los que se produce un cambio para las personas.

\subsection{SUJETOS PARTICIPANTES}

Los y las docentes participantes en el estudio se caracterizan en la siguiente forma:

- 001DocHCSoc: hombre, Ciencias Sociales

- 002DocMCSoc+: mujer, Ciencias Sociales

- 003DocMCEx: mujer, Ciencias Exactas

- 004DocHCEx: hombre, Ciencias Exactas 
- 005DocHCSoc: hombre, Ciencias Sociales

- 006DocHCSoc: hombre, Ciencias Sociales

- 007DocMCEx+: mujer, Ciencias Exactas

- 008DocMCSoc+: mujer, Ciencias Sociales

- 009DocMCSoc: mujer, Ciencias Sociales

- 010DocHCSoc: hombre, Ciencias Sociales

- 011DocHCEx+: hombre, Ciencias Exactas

Todos los sujetos dieron su consentimiento informado para la utilización de la información recopilada en las entrevistas.

Pertenecen a seis facultades diferentes de una universidad privada de Santiago de Chile, que implementa un currículo con enfoque de competencias, y llevan al menos 5 años en la universidad y facultad.

Su participación en procesos de diseño o rediseño ha sido siempre como integrantes de los Comités Curriculares (grupo de docentes que desarrolla y evalúa estos procesos).

Se ha señalado con un signo + a los y las docentes que han tenido cargos de dirección en el proceso.

\subsection{MATERIAL PARA RECOGIDA Y ANÁLISIS DE DATOS}

Corresponde a las textualidades extraídas de 11 entrevistas aplicadas a los y las docentes antes señalados. Estas entrevistas semiestructuradas, abordaron aspectos como su rol en el proceso de rediseño, los elementos que considera más importantes, forma de incidencia en su comprensión de la docencia y la caracterización que hace de una buena práctica docente.

Cabe destacar que los guiones de las entrevistas fueron validados por expertos en el área curricular y de la psicología social. Ello permitió adecuar el guión en forma tal que este se ajustara a los objetivos de la búsqueda de información y a los y las sujetos a los que se aplicarían.

Una vez realizadas las entrevistas se elaboraron matrices de codificación abierta. Tal como lo explican Bonilla-García y López-Suárez (2016), en la codificación abierta se generaron categorías desde la pre-codificación o categorías conceptuales o teóricas (Buendía, Colás y Hernández, 1998). De acuerdo con las mismas autoras, se generaron categorías emergentes o inductivas.

Desde esta codificación abierta se generó una matriz de codificación axial, buscando relaciones entre las categorías y generando familias de categorías y sub-categorías.

Dado que se cuenta con 11 actores entrevistados y considerando que cada uno de ellos/ as vivencia el fenómeno en su propio escenario (no se cuenta con diversidad de actores por escenario), no pareció conveniente generar una nueva matriz para la codificación selectiva. Se establecieron así relaciones conceptuales y teóricas generando algunas categorías eje que permiten comprender la teoría subjetiva que subyace a las textualidades de los/ as entrevistados. Estas proposiciones según Bonilla García y López Suárez (2016) son el enunciado que expresa la relación entre dos o más categorías y sus propiedades.

Es importante señalar que, durante todo el proceso de análisis e interpretación de los datos, el rigor científico fue cautelado siguiendo las indicaciones de Buendía, Colás y Hernández (1998): 
- Veracidad: fue cautelada dado que las entrevistas fueron grabadas y transcritas con el consentimiento de los y las actores.

- Confirmabilidad: todas las codificaciones fueron realizadas en dos fases. En una primera cada uno de los investigadores identificó las categorías. Posteriormente dicha información fue triangulada entre los investigadores.

- Consistencia: dado que se realizó sólo una entrevista, la consistencia fue cautelada triangulando los resultados empíricos con elementos teóricos provenientes de artículos especializados.

\section{RESULTADOS Y DISCUSIÓN}

Para efectos de la selección de categorías relevantes, se consideraron aquellas que aparecieron en las textualidades de al menos 3 actores diferentes.

\subsection{CODIFICACIÓN ABIERTA}

Para analizar la información se consideraron las siguientes definiciones para las categorías teóricas:

- Categoría 1. Insight (INSIGHT): elementos o actividades en el proceso de rediseño que le dieron a la o el entrevistado/a alguna comprensión sobre la práctica docente.

- Categoría 2. Elementos para potenciar práctica docente (ELEM): derivados del proceso de rediseño curricular que la o el entrevistado/a encuentra son importantes para la práctica docente.

- Categoría 3. Rol del docente (ROLDOC): percepción del/a entrevistado/a respecto a las funciones del/a docente en Educación Superior.

- Categoría 4. Creencia en incidencia de la participación (CRETRANS): expresión de la creencia o no creencia (con o sin fundamento) sobre si la participación en el proceso de rediseño tiene incidencia en la práctica docente.

- Categoría 5. Forma de modificación de práctica (MODPRAX): descripción de cómo se modifica la práctica docente a partir de la participación dada.

Respecto a las categorías emergentes:

- Formación (FORM): el o la entrevistado/a señala que la participación en el proceso de diseño curricular se constituye en un espacio de formación que permite la generación de nuevas formas de abordar la docencia y las estrategias didácticas por parte de los/as docentes.

- Diálogo entre colegas (DICOL): el o la entrevistado/a señala que se genera un proceso de comunicación que permite a las y los participantes reflexionar sobre sus prácticas y acceder a nuevas estrategias didácticas que le proponen sus pares.

- Problemas de implementación (IMPLEM): el o la entrevistado/a señala que las exigencias para implementar un proceso curricular enfocado en desarrollo de competencias requieren de recursos humanos y tecnológicos que no siempre están presentes. 
- Resultados de Aprendizajes: (RDA) referido a los logros integrales que los y las estudiantes evidencian y que permiten asegurar el avance en su desarrollo de competencias.

- Experiencia Profesional del/a docente: (EXPRO) aquellas vivencias profesionales de origen, en el terreno laboral que dan al/la docente un aterrizaje claro a sus estrategias didácticas.

- Vacíos en la formación del estudiante: (VACEST) referido a competencias básicas que los y las estudiantes debieran evidenciar al ingreso a la educación superior.

Se presenta a continuación la clasificación de textualidades de las y los entrevistados. Se muestra la categoría teórica, la cantidad de actores que se refirieron a ella (Fr), la totalidad de textualidades que fueron consideradas como aportes a la comprensión de la percepción que se tenía de esta categoría $(\mathrm{Ft})$ y una textualidad que ejemplifica lo planteado por las y los actores:

Tabla 1. Matriz de textualidades, categorías teóricas

\begin{tabular}{|c|c|c|c|}
\hline $\begin{array}{l}\text { Categoría } \\
\text { teórica }\end{array}$ & $\mathbf{F r}$ & $\mathbf{F t}$ & Ejemplo de textualidad \\
\hline INSIGHT & 5 & 25 & $\begin{array}{l}\text { "Al ser partícipe de aquello tú también eres parte de la co-construcción de } \\
\text { este futuro (...profesional), por lo tanto, tu responsabilidad y compromiso } \\
\text { en términos de ser parte de ese sueño, te involucra más" (E5) }\end{array}$ \\
\hline ELEM & 8 & 16 & $\begin{array}{l}\text { "Te permite tener una opinión mucho más activa en torno a cómo } \\
\text { modificar tu asignatura, cómo ver los procesos de otras y considerar } \\
\text { que tu asignatura va más ligada" (E2) }\end{array}$ \\
\hline ROLDOC & 5 & 23 & $\begin{array}{l}\text { "el profesor que no participó en este rediseño y que no sabe lo que } \\
\text { contienen las tripas de esto, no le permite, por ejemplo, hacer un auto- } \\
\text { seguimiento micro-curricular, independiente de la capacitación que se le } \\
\text { haga creo que es muy difícil que cambie su forma de hacer las clases } \\
\text { inmediatamente" }\end{array}$ \\
\hline CRETRANS & 9 & 36 & $\begin{array}{l}\text { "de todas maneras forma a un mejor docente, al ser consciente de todos } \\
\text { esos procesos, tú puedes conducir tu clase de mejor manera y también } \\
\text { con los chiquillos, porque saber para dónde va la micro, de otro lado tú } \\
\text { no eres un engranaje no más, que con un click se prende y se apaga" (E6) }\end{array}$ \\
\hline MODPRAX & 8 & 22 & $\begin{array}{l}\text { "claro y quizás justamente la gente que no tenga tanto tiempo, pero si } \\
\text { me interesaría que participen en el comité curricular porque además } \\
\text { una de las externalidades va a ser que sus clases sean mejores, que } \\
\text { tengan mayor inquietud, interés y empiecen a probar herramientas } \\
\text { nuevas para las clases" (E3) }\end{array}$ \\
\hline
\end{tabular}


A continuación, la matriz que muestra lo ocurrido con las categorías emergentes:

Tabla 2. Matriz de textualidades, categorías emergentes

\begin{tabular}{|c|c|c|c|}
\hline $\begin{array}{l}\text { Categoría } \\
\text { emergente }\end{array}$ & $\mathrm{Fr}$ & Ft & Ejemplo de textualidades \\
\hline $\begin{array}{l}\text { Formación } \\
\text { (FORM) }\end{array}$ & 5 & 12 & $\begin{array}{l}\text { "lo más importante ha sido para mí lidiar con los procesos de } \\
\text { capacitación, porque son a la vez procesos de des - aprendizaje, o } \\
\text { sea, que uno logra que el profesor desarrolle un conjunto de } \\
\text { habilidades pero hay que desmontar en él un conjunto de prejuicios" } \\
\text { (E10) }\end{array}$ \\
\hline $\begin{array}{l}\text { Diálogo entre } \\
\text { colegas } \\
\text { (DICOL) }\end{array}$ & 4 & 9 & $\begin{array}{l}\text { "Aparte de un aprendizaje para los académicos que participamos en } \\
\text { esto, a todos los profesores, incluso a los que lo vieron después, } \\
\text { aunque no los reconozcan les hizo sentido, porque cambiaron su } \\
\text { forma de hacer clases, conversaron sus programas, se motivaron } \\
\text { para hacer este tipo de cosas" (E11) }\end{array}$ \\
\hline $\begin{array}{l}\text { Problemas de } \\
\text { implementación } \\
\text { (IMPLEM) }\end{array}$ & 3 & 6 & $\begin{array}{l}\text { "yo diría que la instalación de los perfiles no fue adecuada, porque } \\
\text { nos fueron impuestos desde arriba y como que nadie entendió } \\
\text { mucho en qué estábamos, nos dieron el perfil y nos obligaron a } \\
\text { buscar las competencias en las asignaturas que ya teníamos" (E3). }\end{array}$ \\
\hline $\begin{array}{l}\text { Resultados de } \\
\text { Aprendizajes } \\
\text { (RDA) }\end{array}$ & 4 & 14 & $\begin{array}{l}\text { "Yo creo que los RDA son lo más importante, porque de alguna } \\
\text { manera, todos hemos manejado programas, y ahí están los objetivos } \\
\text { del programa, los contenidos, pero el tema de pensar que las } \\
\text { asignaturas tienen que obedecer a RDA te cambia la lógica, porque } \\
\text { antes era, pasemos todos estos contenidos, pasemos el programa, la } \\
\text { materia, pero si la lógica es que tenemos que tener un resultado de } \\
\text { aprendizaje, toda la metodología tiene que ir en esa dirección" } \\
\text { (RDA). }\end{array}$ \\
\hline $\begin{array}{l}\text { Experiencia } \\
\text { Profesional del } \\
\text { docente } \\
\text { (EXPRO) }\end{array}$ & 3 & 4 & $\begin{array}{l}\text { "Lo ideal es que el profesor, sobre todo en los primeros años, tenga } \\
\text { que tener esa dualidad trabajo-universidad, cosa que se gane una } \\
\text { experiencia rica, que tu después le puedas transmitir a los cabros" } \\
\text { (E6) }\end{array}$ \\
\hline $\begin{array}{l}\text { Vacíos en la } \\
\text { formación del } \\
\text { estudiante } \\
\text { (VACEST) }\end{array}$ & 5 & 12 & $\begin{array}{l}\text { "La comunidad académica debe estar presente en su totalidad (en el } \\
\text { proceso de rediseño) y por supuesto también los estudiantes, que } \\
\text { tienen mucho que decir porque ellos vienen muy mal formados" } \\
\text { (E5) }\end{array}$ \\
\hline
\end{tabular}

Fuente. Elaboración propia. 
DESARROLLO DOCENTE Y DISEÑO CURRICULAR EN EDUCACIÓN SUPERIOR: UNA SINERGIA NECESARIA PARA MEJORAR LA CALIDAD DE LA EDUCACIÓN

\subsubsection{Mapa relacional de categorías}

Una posible propuesta de relaciones entre las categorías es la que se muestra a continuación:

Tabla 3. Relaciones entre categorías

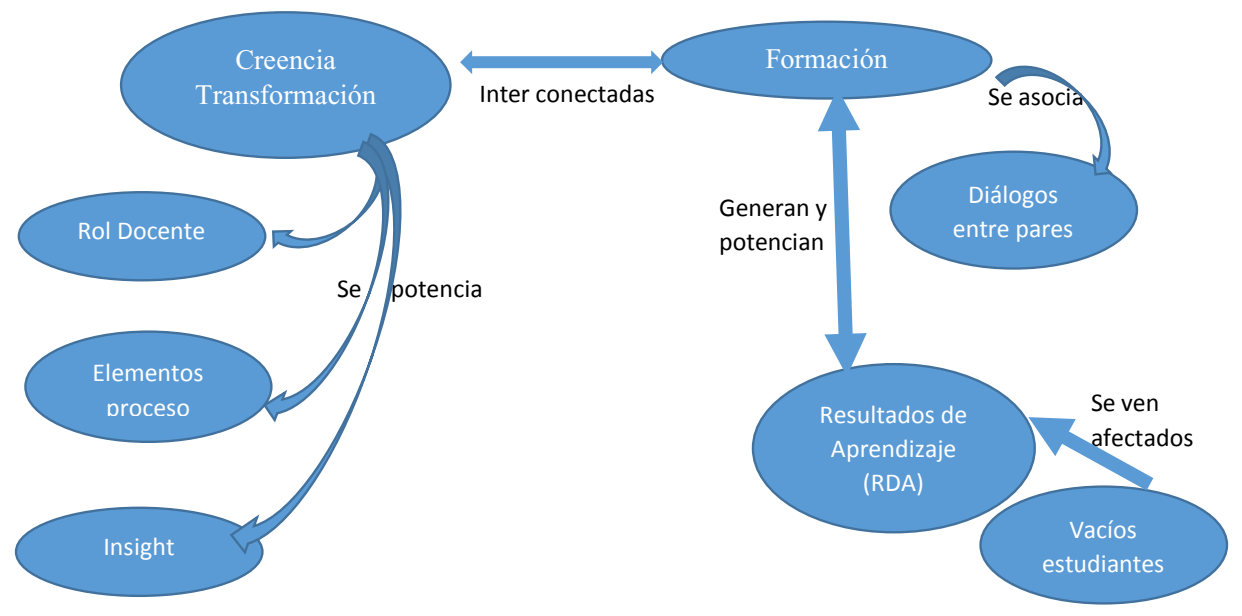

Fuente. Elaboración propia.

\subsection{DESCRIPCIÓN DENSA}

\subsubsection{Proceso de descripción densa}

Clifford Geertz (1983), propone la llamada descripción densa, citando a Gilbert Ryle (1971), quien plantea que esta forma de descripción corresponde a un análisis en el que se indaga en las estructuras de significación, a las que Ryle llamaba códigos establecidos. Geertz (1983) modifica esta definición e incorpora la idea de significación como búsqueda del campo social, lo que permite entender lo que ocurre y sus posibles alcances.

Según Eduardo Weiss (2017), vale más una descripción que devele los hallazgos de los investigadores y las formas en que profundizan en los significados de estos, que las codificaciones y sus tablas que no necesariamente explican con densidad.

4.2.2. Descripción densa del sentido de la participación en el diseño curricular para la práctica docente

\section{- La transformación es posible}

La creencia en la transformación del currículo y su posible impacto en la formación de los y las estudiantes a quienes está dirigido, es sin duda la categoría que con más fuerza es comentada y reflexionada por los y las participantes en este estudio. Se aprecian 36 textualidades distribuidas en la totalidad de los y las participantes. En efecto, se señala con 
fuerza la relevancia de esta participación curricular como motor de transformación del rol del/la docente, tal como lo señala una participante:

ese tipo de cosas yo todas las he aprendido en función de haber estado metida en el tema del desarrollo curricular, de desarrollo del rediseño y esas inquietudes que se han despertado al estar en contacto con estas informaciones y estas capacitaciones que eran necesarias y eso me ha llevado a que haga las cosas distintas en las clases, que haya buscado metodologías diferentes, que me relacione distinto (E003).

Como expresa otro colega, el rol docente cambia no sin dificultad, pero cambia:

Yo viví este cambio cuando empecé a analizar la forma en que hacía el trabajo en sala,... fue con mucha culpa, porque yo tenía la convicción que el profesor se tiene que parar adelante, tiene que dar la información correcta, tiene que manejar toda la información y, el dejar a los chicos trabajando solos y yo sólo apoyando, si bien yo ya había trabajado mucho antes preparando el material, me hacía sentir culpable (...) tenía la sensación de no estar haciendo el trabajo como correspondía (E001).

Estos hallazgos son coincidentes con los de Salazar Botello, Chiang Vega \& Muñoz Jara (2016), quienes destacan la necesidad de potenciar en los y las docentes de educación superior competencias que van más allá de las experticias disciplinares, tales como aquellas que se relacionan con interacciones apropiadas y generación de climas de aula motivantes, desafiantes pero acogedores, exponiendo así un rol docente complejo para el que todos y todas debemos prepararnos. En la misma forma, y relacionando esta forma de asumir el rol docente con los aspectos curriculares, Veiravé y Ojeda (2003) unen la participación del /la docente en la transformación del currículo con el rol docente, como formas fundamentales para potenciar la real calidad educativa. En el estudio, docentes de áreas disciplinares muy diversas reconocen esta necesidad.

Los elementos específicos del proceso curricular aparecen como relevantes. Así, un docente expresa, al referirse a la forma en que se ha procedido en la reformulación del currículo, para adecuarlo a un enfoque de competencias, que:

yo creo que hay alli competencias que debiesen ser transversales a toda asignatura, siempre pensando en vincularlo con la realidad, porque si no, finalmente pasa lo que nos pasó a muchos de los que venimos de la vieja escuela, que tenemos mucha información, mucha memorización, pero que no teníamos mucho contacto con la realidad (E005).

Imbernon \& Guerrero (2017), destacan esta aparición de currículos sustentados en competencias, que se hacen cargo en alguna forma de la necesidad de conectar fuertemente la realidad con las disciplinas, en forma tal que estos nuevos estudiantes que llegan a la educación superior se sientan acogidos en su nueva realidad tecnológica e interconectada. Respecto a esta posibilidad transformadora, los/las docentes señalan que es este involucramiento con las diversas etapas del proceso lo que permite generar comprensiones (insight). Una docente participante señala claramente esta mirada profunda al referirse a la transformación que la toma de conciencia de lo relevante del proceso genera: 
fue un proceso no solamente valorado por los del comité, sino que fue valorado por todos los colegas, al ver el tiempo que nos dimos para juntarnos, al conversar con nosotros más abiertamente y con más holgura, de procesos y decisiones que sin duda alguna nos cambiaron la mirada (E002).

De la misma forma, otro docente plantea que: "al ser partícipe de aquello tú también eres parte de la co-construcción de este futuro (...profesional), por lo tanto, tu responsabilidad y compromiso en términos de ser parte de ese sueño, te involucra más" (E005); se aprecia así su compromiso profundo con lo que se estaba construyendo. Este insight nos parece, y se confirma con lo expresado por los y las docentes entrevistados/as, es coherente con los planteamientos de Salas (2016), al hacer referencia a la evaluación permanente del currículo desde su primera elaboración, para asegurarse del cumplimiento de su objetivo central: la formación humana. El diseño del currículo establece cuál es el tipo de persona que se desea formar y la sociedad que se desea colaborar a construir, y toda la propuesta curricular busca ofrecer una formación para los y las estudiantes que incorpore su vida plena y su inserción crítica en la sociedad. Y es en estos aspectos profundos en los que el insight se apoya para generarse; como expresaba el docente antes citado, es la "responsabilidad y compromiso en términos de ser parte de ese sueño". Nos parece que es aquí donde los y las docentes, con o sin plena conciencia de ello, aluden a los aspectos políticos del currículo. Es este aspecto político de todo proceso de construcción social, evidenciado en su sustento teórico e ideológico (Apple, 2012, 2019; Freire, 1958), lo que las y los docentes sienten que ellas y ellos tienen la opción de definir, de intervenir y transformar, y esto lo aprecian y valoran; los compromete con esos fines y estrategias que ellas y ellos han podido proponer.

\section{- Formarse para formar}

Se ha determinado como categoría emergente que un número importante de textualidades (12) de diversos/as participantes (8) destacan, la formación del y la docente de educación superior, proceso que se ve enriquecido con la participación en el proceso curricular. Uno de los docentes entrevistados, al referirse a este aspecto plantea que se hace necesario: "instalar otros procesos en el mismo sistema educacional, comunidades de aprendizaje, procesos de autoevaluación, hay que levantar una cultura con un enfoque curricular mucho más dialéctico ... que permita que nos perfeccionemos" (E005) y el docente insiste que ello puede o debe ocurrir en el mismo proceso de implementar el currículo. Destacando esta necesidad, una docente explica que se requiere que el o la docente de educación superior sea:

alguien que esté actualizado, que esté leyendo, que esté incorporado al avance ... por un lado en la metodología de enseñanza y por otro lado en la disciplina, y por otro lado en el estudiante, que es un adulto, yo creo que también tiene que tener una formación el profesor (E003).

En efecto estas ideas son coherentes con el llamado desarrollo profesional docente, o formación profesional docente, todo ello a nivel de educación terciaria. Villalobos y Melo (2008) y Aramburuzavala, Hernández-Castilla \& Uribe (2013), exponen que este es un proceso insoslayable puesto que incide en la calidad de la formación de las y los estudiantes. Sostienen que este proceso ha de enfatizar las competencias educacionales y, 
tal como plantean Imbernon \& Guerrero (2017), han de buscar una formación integral y crítica de los y las estudiantes mediante adecuadas mediaciones, planificaciones didácticas que acogen la diversidad y trabajos en equipo y contextualizados adecuadamente. Esto significa romper con la clase tradicional, homogeneizante y abstracta. Un docente expresa esto en los siguientes términos:

lo más importante ha sido para mí lidiar con los procesos de capacitación en este nuevo enfoque (de competencias integrales y contextualizadas), porque son a la vez procesos de des-aprendizaje, o sea, que uno logra desarrollar... un conjunto de habilidades, pero hay que desmontar... un conjunto de prejuicios (E010).

Y sin duda que esta formación sólo es real cuando transforma desde la profundidad de las creencias, de las convicciones; en este sentido, los y las docentes destacan la relevancia del diálogo reflexivo entre colegas como un potente factor formador y, por ende, transformador.

Esta evaluación permanente que se debe hacer...en este proceso curricular, en la primera instancia logras tener una mirada general articulada; luego de aquello viene la mirada operativa, y allí los docentes, una vez en el aula, ya frente a la asignatura, empiezan a ver otros ajustes que implican también una reflexión potente y es en ese minuto donde las reuniones y los diálogos resultan lo más operativo (E002).

Este docente enfatiza la necesidad de este diálogo entre pares en el marco del desarrollo y evaluación curricular, para generar reflexiones verdaderamente profundas y transformadoras. Respecto a esto no se han encontrado estudios que aludan específicamente al diálogo reflexivo en torno a las prácticas. Aunque sin duda las llamadas comunidades de aprendizaje están en esta línea.

\section{- Los resultados del aprendizaje en un enfoque de competencias}

Esta categoría emergente resulta para los y las docentes de enorme relevancia para varios de ellos y ellas; 14 textualidades distribuidas en 6 docentes permiten suponer que se encuentra aquí un sentido relevante del desarrollo docente que la participación en un proceso curricular genera. Es interesante destacar que los resultados de aprendizaje, tal como se señala en el manual de apoyo docente "Evaluación para el aprendizaje" (2017), se consideran como la descripción de aquello que se espera que los y las estudiantes sepan, comprendan y demuestren al finalizar una unidad o módulo. Este resultado debe explicitar qué debe hacer o aplicar el o la estudiante, sobre qué elementos o aspectos debe aplicar este saber, con qué fundamentos toma las decisiones, en qué contexto aplica (con quiénes, dónde) y para qué lo hace (la finalidad). Se aprecia que en este manual el resultado de aprendizaje posee los elementos de una competencia integral o compleja y que se acepta que la competencia es más holística y abarcadora, insertándose en ella un conjunto de resultados de aprendizaje. De la Fuente, Ross, Ferrer, Muñoz, Cavas y Ross (2014), citando a López Camps (2005) y García Martín et al. (2012), consideran que los resultados esperados del aprendizaje están referidos al comportamiento o acción observable del/la estudiante al finalizar el proceso de aprendizaje. No se especifica en este trabajo el carácter integral que se aprecia en el manual 
de evaluación antes citado. Los y las docentes entrevistados aluden a este sentido complejo, cuando por ejemplo uno de ellos expresa que:

una parte muy importante son los resultados de aprendizaje, o sea sacarse el chip del objetivo y cambiarlo por este resultado de aprendizaje (RDA) y que no sea típico capacitar al alumno para...eso ya no corre. Algunos dicen que es lo mismo, pero no es lo mismo, en el objetivo importa el contenido, el RDA es más complejo y por tanto no puedes hacer la misma prueba que antes (E011).

De una u otra forma las textualidades de los y las docentes aluden a la relevancia de estos RDA, entendidos de esta forma integral y compleja. Una docente expresa que

...los RDA son lo más importante, porque de alguna manera, todos hemos manejado programas, y ahí están los objetivos del programa, los contenidos, pero el tema de pensar que la asignatura tiene que obedecer a RDA te cambia la lógica, porque antes era, pasemos todos estos contenidos, pasemos el programa, la materia, pero si la lógica es que tenemos que tener un resultado de aprendizaje, toda la metodología tiene que ir en esa dirección (E009).

El diseño curricular les exige modificar su perspectiva y concuerdan que esta participación en el proceso de diseño o rediseño ha permitido un cambio real y profundo.

\section{- Dificultades: los vacíos en la formación previa de los y las estudiantes}

Esta categoría emergente está referida a las dificultades que presentan los y las estudiantes en el proceso formativo debido a vacíos que se presentan en diversos ámbitos. Algunos docentes plantean que no siempre resulta sencilla la tarea formativa debido a vacíos cognitivos e incluso actitudinales con que los y las estudiantes ingresan a la educación superior. Así, un docente plantea que: "los alumnos que llegan a la universidad muchas veces están desprovistos de capital cultural de manera bastante dramática y entonces ahí hay que poner mucho acento digamos en tratar de alguna manera de paliar las deficiencias que vienen desde la escuela" (E009). El docente plantea que en esta forma se pierde tiempo en lograr que los y las estudiantes superen sus dificultades y puedan proceder a aprender en forma profunda y comprensiva.

El vacío no sólo parece ser siempre cognitivo; en ocasiones se aprecian dificultades motivacionales, que los/las llevan a desertar o a ocupar su tiempo en acciones que no le permiten lograr los aprendizajes exigidos en su formación. Una de las docentes entrevistadas sostiene que: "el estudiante de primer año que recién está ingresando, lo que más necesita es que le refuercen su motivación" (E007). Ella considera que la acogida del estudiante como persona integral es fundamental para invitar al estudiante a aprender con sentido y motivadamente.

Algunos/as docentes creen que estos vacíos pueden superarse y que ello depende de que encontremos estrategias necesarias para formar personas integrales y laboralmente eficientes; estas estrategias, coherentes con el currículo construido, han de buscar el desarrollo de las competencias complejas propuestas, mediante estrategias activas, auténticamente aplicadas a situaciones laborales: 
Al principio los estudiantes reclamaban que era mucho trabajo pero una vez que van viendo el resultado y ven que su aprendizaje es vivencial y que no se les va a olvidar nunca más el asunto cambia y aprenden a ser más autónomos y las evaluaciones son buenas (E011).

Antonio Aguilera Jiménez (2000), expone claramente la necesidad de una formación universitaria que se haga cargo de la formación de competencias complejas y aplicadas que dejen fuera los contenidos innecesarios y excesivos. Agrega que la formación terciaria, específicamente universitaria busca formar profesionales, investigadores que complementen permanentemente su formación y ciudadanos que aporten a su sociedad. Pinto \& Cortés (2017), sostienen que la educación superior que busca formar profesionales ha de potenciar como competencia transversal el aprendizaje estratégico que permite que los y las estudiantes generen aprendizajes autónomos en el futuro, mediante diversas estrategias de investigación aplicada. Pero esta competencia transversal, según plantean algunos de los entrevistados, no resulta posible, puesto que los y las estudiantes son:

gente extraordinariamente poco dispuesta incluso diría con dificultades de comprensión muy seria y de discusión y ver las cosas desde una óptica podríamos decir debatibles o dialéctica, crítica... no, no son capaces de ponerse en esa posición están esperando que uno le enseñe las fórmulas (E009).

Yolanda Argudín (2001) destaca la necesidad de considerar en la formación terciaria, cuando es enfocada desde las competencias, elementos conceptuales, procedimentales y valóricos. Así, la ciudadanía que involucra un conjunto relevante de valores de participación, solidaridad y justicia, entre otros, debe también ser potenciada, aspecto que, según los y las docentes entrevistados no siempre es sencillo por el vacío que hay a nivel escolar en la formación ciudadana: "desde el liceo... sobre todo en materias de lo que se entendía antes por educación cívica” (E009).

\section{CONCLUSIONES Y DISCUSIÓN}

El presente trabajo muestra cómo la participación en el comité curricular de una carrera puede ser considerado como espacio de desarrollo docente, donde los y las profesores/as son capaces de encontrar sentido a su tarea educativa a partir de la experiencia obtenida del análisis del diseño o rediseño curricular de una carrera.

El hecho de poder analizar la estructura curricular en su totalidad parece entregar una visión de conjunto que les permite ubicar la contribución de su asignatura desde un punto de vista sistémico. Esto conlleva a que los y las docentes tengan elementos para poder modificar o potenciar su práctica educativa, probando, por ejemplo, nuevas metodologías de enseñanza-aprendizaje. Por otra parte, los resultados de aprendizaje (RDA) definidos en la asignatura toman otro cariz a la luz de esta mirada global del proceso formativo.

En relación al espacio mismo de desarrollo docente, las entrevistas nos permiten identificar una componente de transformación de las creencias docentes, particularmente a través de un punto de vista distinto respecto al rol docente, lo que permite una nueva perspectiva, siempre en el marco de la comprensión global del proceso formativo que 
entrega la participación en el comité curricular. Es posible identificar una componente asociada a la formación, donde el diálogo entre pares y el análisis de los resultados de aprendizaje de las asignaturas son centrales. Así, el proceso de diseño/rediseño curricular se constituye en un espacio de hetero-formación (de la parte de los asesores curriculares que acompañan el proceso), de co-formación (entre los pares miembros del comité) de auto-formación (de investigación personal, respecto a temas educativos y curriculares).

Sin embargo, ¿es posible que todas las y las docentes de una determinada titulación o carrera participen en procesos de diseño/rediseño curricular? Dadas las restricciones del trabajo (tiempo, horarios, recursos materiales, etc.) pareciera ser que esto no es posible. Es necesario idear entonces un sistema que permita a todos los y las docentes beneficiarse de la comprensión que esto otorga, a través de distintos grados de involucramiento en el proceso, donde es claro que los participantes del comité deberán actuar como formadores de formadores. El diseño de este sistema deberá ser un aspecto fundamental de un proceso global de desarrollo docente al interior de una institución.

\section{REFERENCIAS BIBLIOGRÁFICAS}

Abbagnano, N. y Visalberghi, A. (2016). Historia de la pedagogía. México: Fondo de Cultura Económica. Aguilera Jiménez, A. (2000). Formación universitaria en la sociedad de la información. Escuela Abierta, (4), 69-84.

Apple, M. (2012). Education and power. Nueva York: Routledge. . (2019). Ideology and curriculum. Nueva York: Routledge.

Aramburuzavala, P., Hernández-Castilla, A. \& Uribe, I. (2013). Modelos y tendencias de la formación docente universitaria. Revista de Currículum y formación del profesorado, 17(3), 345-357.

Argudín, Y. (2001). Educación basada en competencias. Magistralis, (20), 39-61.

Arteaga, J. (2015). Algunas reflexiones en torno al perfeccionamiento del diseño curricular. Revista Electrónica Formación y Calidad Educativa (REFCalE), 151-160.

Bayer, A. (2007). Universidad versus academia: la esencia del quehacer universitario. Ánfora año 14, (22), Universidad de Manizales, 157-179.

Bonilla-García, M. \& López-Suárez, A. (2016). Ejemplificación del proceso metodológico de la teoría fundamentada. Cinta de moebio 57, 305-315.

Buendía, L., Colás, P. y Hernández, F. (1998). Métodos de Investigación en Psicopedagogía. Madrid: Mc Graw Hill.

Casanova, A. M. (2012). El diseño curricular como factor de la calidad educativa. Revista iberoamericana sobre calidad, eficacia y cambio en educación, 7-20.

Castro Estrada, M. L., Rodríguez Rejas, M. J. y Urteaga Urías, E. (2016). Abrir las aulas: el vínculo entre docencia, investigación y vinculación comunitaria. Revista Brasilera de Educación. Rio de Janeiro 21(66), 737-758,

Chalmers, D. \& Garnier, D. (2015). The measurements and impact of university teacher development programs. Educar, 51(1), 53-80.

Comenius, J. A. (2012). Didáctica magna. Madrid: AKAL.

De la Fuente, M., Ross, D., Ferrer, M., Muñoz, M., Cavas, F. \& Ross, L. (2014). La relación de los resultados de aprendizaje, la metoología docente y la metodología de evaluación. Alicante, España: Universidad de Alicante, vicerrectorado de Estudios, Formación y calidad.

Dussel, I. (2001). La formación de docentes para la educación secundaria en América Latina: perspectivas comparadas. En C. D. Braslavsky, Los formadores de jóvenes en América latina. Desafios, experiencias y propuestas. (págs. 10-23). Ginebra: Oficina Internacional de educación y administración nacional de educación pública Uruguay. 
Evans, L. (2002). What is teacher development? Educar 51(1), 53-80.

Fonseca Pérez, J. \& Gamboa, M. (2017). Aspectos teóricos sobre el diseño curricular y sus particularidades en las ciencias. Revista Boletín Redipe 6(3), 83-112.

Freire, P. (1958). Pedagogía del Oprimido. Ciudad de México: Siglo XX editores.

Geertz, C. (1983). Descripción densa: hacia una teoría interpretativa de la cultura. Barcelona: Gedisa.

Gibbs, G. \& Coffer, M. (2004). The impact of training of university teachers on their teacher's skills, their approach to teaching and the approach to learning of their students. Active Learning in Higher Eduction 5(1), 87-100.

González, L. y Espinoza, Ó. (2008). Calidad de la educación superior: conceptos y modelos. Revista Calidad en la Educación CNED, (28), 248-276.

González Maura, V. \& López Rodríguez, A. y. (2017). Diplomado en docencia e investigación universitaria. Revista Iberoamericana de Educación - OEI/CAEU, 121-146.

Habl, J. (2017). On Being Human(e): Comenius' Pedagogical Humanization as an Anthropological Problem. Oregon: Pickwick Publications

Herbart, J. (2013). Dos escritos sobre educación. Santiago: Peuma Ediciones.

Icarte, G. A. y Labate, H. A. (2016). Metodología para revisar y actualizar el diseño curricular de una carrera universitaria que incorpora el concepto de aprendizaje basado en competencias. Formación universitaria, 9(2), 03-16.

Imbernon, F. \& Guerrero, C. (2017). ¿Existe en la universidad una profesionalización docente? Revista de Educación a Distancia 56, 1-12.

Navarro, G., Varas, M., González, M. \& Catalán, R. (2017). Percepción de académicos sobre efectos a nivel personal e institucional de su participación en un programa de formación para desarrollar competencias genéricas en la Universidad de Concepción. Revista de Estudios y Experiencias en Educación Vol.16 Num. 30, 49-61.

OECD. (2005). Teachers matter: attracting, developing and retaining effective teachers. París: OECD.

Osorio, M. (2017). El currículo: perspectivas para acercarnos a su comprensión. Zona próxima: revista del Instituto de Estudios Superiores en Educación, (26), 140-151.

Pestalozzi, J. H. (2003). El canto del cisne. Barcelona: Laertes.

Pinto, A. \& Cortés, O. (2017). ¿Qué piensan los estudiantes universitarios frente a la formación investigativa? Revista Docente Universitaria, 57-75.

Salas Perea, R. (2016). Is curriculum redesigning without previous curricular assessment scientific? Educación Médica Superior, 30(2), 1-11.

Salazar Botello, C. M., Chiang Vega, M. M. \& Muñoz Jara, Y. (2016). Competencias docentes en la educación superior: un estudio empírico en la universidad del Bio Bio. Revista Electrónica "Actualidades Investigativas en Educación”, 1-28.

Strauss, G. y Corbin, J. (2002). Bases de la investigación cualitativa. Antioquia, Colombia: Universidad de Antioquia.

Veiravé, D. y Ojeda, M. (2003). Diseño y desarrollo curricular, una tarea docente. Revista Nordeste, 153-161.

Villalobos, A. \& Melo, Y. (2008). La formación del profesor universitario: Aportes para su discusión. Universidades (México, DF), (39), 3-20.

Vivar, C. G., McQueen, A., Whyte, D. A. \& Canga, N. (2013). Primeros pasos en la investigación cualitativa: desarrollo de una propuesta de investigación. Index de Enfermería, 22(4), 222-227. https://dx.doi.org/10.4321/S1132-12962013000300007.

Vivar, C. G., Arantzamendi, M, López-Dicastillo, O. \& Gordo, L. (2010). La Teoría Fundamentada como Metodología de Investigación Cualitativa en Enfermería. Index de Enfermería, 19(4), 283-288. http:// scielo.isciii.es/scielo.php?script=sci_arttext\&pid=S1132-12962010000300011\&lng=es\&tlng=es.

Weiss, E. (2017). Hermenéutica y descripción densa versus teoría fundamentada. RMIE, 22(73) (ISSN: 14056666), 637-654. 
